
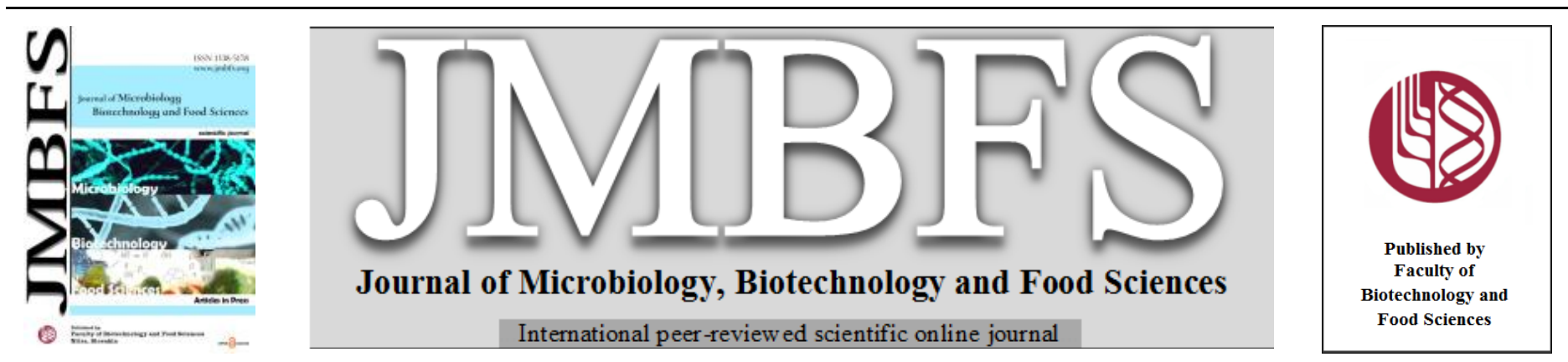

\title{
ASSOCIATION BETWEEN SINGLE AND MULTIPLE ELECTRICAL CONDUCTIVITY MEASUREMENTS AND PORK QUALITY ATTRIBUTES
}

\author{
Katarzyna Antosik ${ }^{1}$, Krystian Tarczyński*2, Elżbieta Krzęcio-Nieczyporuk ${ }^{1}$, Halina Sieczkowska ${ }^{2}$, Andrzej Zybert ${ }^{2}$ \\ Address(es): Krystian Tarczyński, PhD \\ ${ }^{1}$ Siedlce University of Natural Sciences and Humanities, Faculty of Medical and Health Sciences, Prusa 14 Street, $08-110$ Siedlce, Poland. +48 25 643 1362. \\ ${ }^{2}$ Siedlce University of Natural Sciences and Humanities, Faculty of Agrobioengineering and Animal Husbandry, Prusa 14 Street, 08-110 Siedlce, Poland.
}

*Corresponding author: krystian.tarczynski@uph.edu.pl

https://doi.org/10.55251/jmbfs.3805

\section{ARTICLE INFO}

Received 7. 10. 2020

Revised 20. 1. 2022

Accepted 20. 1. 2022

Published 1.6. 2022

Regular article

open $O$ access

\begin{abstract}
The aim of this study was to determine the usefulness of single and multiple electrical conductivity measurements done directly in abattoir in pork quality attributes evaluation. 82 commercial hybrid fatteners ( 41 barrows and 41 gilts) originated from the same breeder and reared under the same environmental conditions were slaughtered at approximately $110 \mathrm{~kg}$ live weight. Electrical conductivity was measured at 35 min. $\left(\mathrm{EC}_{35}\right)$ and $2\left(\mathrm{EC}_{2}\right), 3\left(\mathrm{EC}_{3}\right)$ and 24 hours post mortem $\left(\mathrm{EC}_{24}\right)$. Pork quality was characterised after slaughter by meat acidity (pH) measured at $35 \mathrm{~min}$. and 24 hours, meat colour parameters (CIE-L*a* ${ }^{*}{ }^{*}$ system) at $24 \mathrm{~h}$, drip loss at $48 \mathrm{~h}\left(\mathrm{DL}_{48}\right), 96 \mathrm{~h}(\mathrm{DL} 96)$ and $144 \mathrm{~h}$ $\left(\mathrm{DL}_{144}\right)$, water holding capacity (WHC) and tenderness at $48 \mathrm{~h}$ and $144 \mathrm{~h}$ expressed by shear force $\left(\mathrm{N} / \mathrm{cm}^{2}\right)$. Electrical conductivity was rather weakly correlated with pork quality traits (the highest Pearson's r, e.g. 0.30 was noted both for $\mathrm{EC}_{2}$ and a and DL 48 ). Highest $\mathrm{C}_{\mathrm{r}}$ and $\mathrm{C}_{\mathrm{r}}^{2}$ values for single and multiple electrical conductivity measurements and sets of pork quality traits were noted for drip loss and water holding capacity $\left(0.42^{* *}\right.$ and 0.29 and $0.57^{* *}$ and 0.32 respectively) while for most of remaining sets no statistical significance were found. It could be presumed then that electrical conductivity are not useful predictor of pork quality attributes that could be performed directly in abattoirs (besides drip loss and water holding capacity to some extent). Therefore it is suggested to revise this parameter use in pork quality evaluation however further examination covering larger population of fatteners should be performed.
\end{abstract}

Keywords: pork, fatteners, electrical conductivity

\section{INTRODUCTION}

For several decades pig breeding strategies were oriented directly on production of lean and fast growing fatteners which contributed to deterioration in pork quality (Lonergan et al., 2001, Dokmanović et al., 2015). However recent consumers demands of pork/pork products quality and uniformity are constantly increasing and became a major concern for meat industry (Ngapo et al., 2007). As a result, some countries introduced high quality pork production systems and chains that matches consumer demands in $21^{\text {st }}$ century (Verbeke et al., 2010; Grunert et al. 2011; Font-i-Furnols \& Guerrero, 2014). The rise of consumers demands also imposed pork industry to perform pork quality evaluation on a continuous basis where electrical conductivity (EC) is of high interest due to its ability of direct use in abattoirs (Morel et al., 2006; Mörlein et al., 2007b; Jukna et al., 2012; Karamucki et al., 2015). In meat, direct electrical current can flow only around cells due to insulation of plasma membranes. However, when cell membranes are permeable (for instance by protein denaturation) electrical current can also flow through the interior of the cells (fluid can move between intracellular and extracellular spaces). That increase electrical conductivity which is governed mainly by small ions, e.g. $\mathrm{Cl}^{-}, \mathrm{K}^{+}$and $\mathrm{Na}^{+}$and therefore indication of cell membranes integrity is possible. Enhanced electrical conductivity can then be used as an indicator of drip loss and tenderness of meat (Pliquett et al., 1990; 2003). Although electrical conductivity is widely used as pork quality predictor there is still not enough information in literature about its measuring efficiency. Moreover, discordant opinions about its usefulness are noted and sparse knowledge about the potential of multiple measurements is available (Lee et al., 2000; Morel et al., 2006; Mörlein et al., 2007b; Czyżak-Runowska et al., 2010; Jukna et al., 2012). The aim of this study was to determine the usefulness of single and multiple electrical conductivity measurements in the abattoir at 35 minutes, 2, 3 and 24 hours post mortem in pork quality attributes evaluation.

\section{MATERIAL AND METHODS}

Animals rearing, transport and slaughter
The study was carried out in autumn on 82 commercial hybrid fatteners (41 barrows and 41 gilts) originated from the same breeder. Animals were characterised by similar live mass (ca. $110 \mathrm{~kg}$ ) and slaughter age (ca. 160 days). Environmental conditions were the same for all animals throughout the rearing (concrete floor). Fatteners were fed complete feed mixtures according to age (12$13 \mathrm{MJ}$ ME). The animals were transported in specialized pig transport vehicles to slaughterhouse (c.a. $300 \mathrm{~km}$ ) after loading by qualified personnel without using of electrical pods. The fatteners were slaughtered 2-4 hours after transportation using an electric stunner INARCO Dutch line $(250 \mathrm{~V}, 1.5 \mathrm{~s})$ and bled lying down in accordance with the procedure accepted at the abattoir. Lean meat content was determined $35 \mathrm{~min}$. post mortem by ULTRA-FOM 300 (SFK-Technology, Denmark) and hot carcass weight (HCW) was measured immediately afterwards (accuracy up to $0.1 \mathrm{~kg}$ ). Next, carcasses were chilled in three-phase chilling tunnel $\left(-10^{\circ} \mathrm{C}\right.$ for $15 \mathrm{~min} .,-15^{\circ} \mathrm{C}$ for $25 \mathrm{~min}$. and $-5^{\circ} \mathrm{C}$ for $40 \mathrm{~min}$. with air velocity of 3 $\mathrm{m} / \mathrm{s}$ ) and stored at $4^{\circ} \mathrm{C}$ up to $24 \mathrm{~h}$ after the slaughter.

\section{Meat quality measurements}

Electrical conductivity was measured directly on the hanging carcasses in the Longissimus dorsi muscle (Longissimus lumborum - LL, behind the last rib) 35 min $\left(\mathrm{EC}_{35}\right), 2$ hours $\left(\mathrm{EC}_{2}\right), 3$ hours $\left(\mathrm{EC}_{3}\right)$ and 24 hours $\left(\mathrm{EC}_{24}\right)$ post mortem using the LF-Star (Matthaüs, Germany) apparatus with a frequency of $1.2 \mathrm{kHz}$ and automatic temperature compensation. Acidity of the muscle tissue $(\mathrm{pH})$ was measured directly in hanging carcasses $35 \mathrm{~min}\left(\mathrm{pH}_{1}\right)$ and 24 hours $\left(\mathrm{pH}_{24}\right)$ post mortem using a calibrated pistol pH-meter MASTER (Draminski, Olsztyn, Poland) with temperature compensation. The electrodes were placed crosswise to the muscle fibres. The samples for assessment of remaining parameters were separated from the bone, external fat and epimysium and then stored in plastic bags at $0-4^{\circ} \mathrm{C}$ Meat colour light reflectance scores for CIE L ${ }^{*} a^{*} b^{*}$ (Commission Internationale de l'Eclairage, 2007) were assessed $24 \mathrm{~h}$ post mortem using a Minolta CR310 apparatus (Japan) $24 \mathrm{~h}$ post mortem calibrated against the white plate (iluminat D65 and $10^{\circ}$ standard observer) . Drip loss was assessed $48 \mathrm{~h}\left(\mathrm{DL}_{48}\right), 96 \mathrm{~h}\left(\mathrm{DL}_{96}\right)$ and $144 \mathrm{~h}\left(\mathrm{DL}_{144}\right)$ according to Prange et al. (1977). Water holding capacity (WHC) was determined with the filter paper method according to the method of Grau \& Hamm (1952) as modified by Pohja \& Ninivaara (1957). Tenderness of 
meat was determined at $48 \mathrm{~h}$ and $144 \mathrm{~h}$ post mortem and expressed by shear force $\left(\mathrm{N} / \mathrm{cm}^{2}\right)$ using an Instron 1140 (USA) apparatus with Warner - Bratzler device according to PN-ISO Norm 11036:1999.

Experiment layout

Firstly, phenotypic correlations (Pearson's r) between single electrical conductivity measurements and particular pork quality traits and canonica correlation were examined. Then canonical correlation analysis has been used to evaluate the relationships between single and multiple electrical conductivity measurements and pork quality attributes sets. Canonical correlation analysis is a generalization of multiple regression analysis with more than one set of dependent variables. It allows to determine how much of variance of one set is predictable by the other by quantifying the common variance between them (maximum correlation between variables is estimated by linear combinations). The correlations are expressed by canonical correlation $\left(\mathrm{C}_{\mathrm{r}}\right)$. Squared canonical correlation $\left(\mathrm{C}_{\mathrm{r}}^{2}\right)$ represent the amount of variance in one canonical variate that is accounted for by the other (Weiss, 1972). Therefore, the aim of canonical correlation analysis is to estimate square canonical correlation when the canonical correlation is maximum. In present study eight sets of independent variables (single and multiple measurements of electrical conductivity), e.g. $\mathrm{X}_{1}-\mathrm{EC}_{35} ; \mathrm{X}_{2}-$ $\mathrm{EC}_{2} ; \mathrm{X}_{3}-\mathrm{EC}_{3} ; \mathrm{X}_{4}-\mathrm{EC}_{24} ; \mathrm{X}_{5}-\mathrm{EC}_{35}, \mathrm{EC}_{2} ; \mathrm{X}_{6}-\mathrm{EC}_{35}, \mathrm{EC}_{3} ; \mathrm{X}_{7}-\mathrm{EC}_{2}, \mathrm{EC}_{3} ; \mathrm{X}_{8}-$ $\mathrm{EC}_{35}, \mathrm{EC}_{2}, \mathrm{EC}_{3}$ and ten sets of dependent variables (pork quality attributes), e.g.

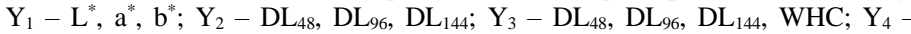
$\mathrm{WBSF}_{48}, \mathrm{WBSF}_{144} ; \mathrm{Y}_{5}-\mathrm{DL}_{48}, \mathrm{WBSF}_{48} ; \mathrm{Y}_{6}-\mathrm{DL}_{48}, \mathrm{WHC}, \mathrm{WBSF}_{48} ; \mathrm{Y}_{7}-\mathrm{DL}_{48}$ $\mathrm{WHC}_{\text {, }} \mathrm{WBSF}_{48}, \mathrm{~L}^{*} ; \mathrm{Y}_{8}-\mathrm{DL}_{48}, \mathrm{WHC}, \mathrm{TY} ; \mathrm{Y}_{9}-\mathrm{DL}_{48}, \mathrm{WHC}, \mathrm{TY}, \mathrm{L}^{*} ; \mathrm{Y}_{10}-\mathrm{DL}_{48}$, WHC, TY, L*, $\mathrm{WBSF}_{48}$ were analysed. Additionally an examination of the correlation between original variables and their related canonical variables was performed. Canonical variables (weighted average of the variables of two sets) are considered as implicit variables (U and V) that are synthetic index (rate) defining the correlation between both sets.

\section{Statistical analysis}

Both phenotypic correlation coefficients (Pearson's r) and canonical correlation analysis were performed by the use of STATISTICA 13.5 (StatSoft, Tulsa, OK USA) software. Besides mean values and standard deviations $( \pm \mathrm{SD})$, minimal and maximal values and respective coefficients of variances $(\mathrm{CV})$ for all measured traits were presented.

\section{RESULTS AND DISCUSSION}

All research material was characterised by hot carcass weight of $85.27 \mathrm{~kg} \pm 5.66 \mathrm{~kg}$ and lean meat content of $56.96 \% \pm 4.53 \%$. Electrical conductivity values measured up to 24 hours post mortem and meat quality traits values of analysed population were shown in Table 1 and 2 respectively.

Table 1 Electrical conductivity measured up to 24 hour post mortem

\begin{tabular}{lccccc} 
Trait & Min. & Max. & Mean & SD & CV [\%] \\
& & & & & \\
\hline $\mathrm{EC}_{35}[\mathrm{mS} / \mathrm{cm}]$ & 2.40 & 11.70 & 3.89 & 1.53 & 39.20 \\
$\mathrm{EC}_{2}[\mathrm{mS} / \mathrm{cm}]$ & 1.50 & 12.50 & 3.23 & 1.68 & 52.01 \\
$\mathrm{EC}_{3}[\mathrm{mS} / \mathrm{cm}]$ & 1.30 & 12.90 & 4.10 & 2.08 & 50.64 \\
$\mathrm{EC}_{24}[\mathrm{mS} / \mathrm{cm}]$ & 1.50 & 11.20 & 4.94 & 1.75 & 35.41 \\
\hline
\end{tabular}

Table 2 Meat quality traits of analysed population

\begin{tabular}{lccccc} 
Trait & Min. & Max. & Mean & SD & CV [\%] \\
& & & & & \\
\hline $\mathrm{pH}_{1}$ & 5.82 & 6.90 & 6.48 & 0.24 & 3.75 \\
$\mathrm{pH}_{24}$ & 5.48 & 6.07 & 5.68 & 0.13 & 2.23 \\
$\mathrm{~L}^{*}$ & 48.45 & 68.66 & 55.65 & 4.36 & 7.09 \\
$\mathrm{a}^{*}$ & 11.87 & 17.98 & 14.56 & 1.33 & 8.41 \\
$\mathrm{~b}^{*}$ & 3.03 & 9.97 & 5.77 & 1.61 & 25.37 \\
${\mathrm{WHC}\left[\mathrm{cm}^{2}\right]}_{\mathrm{DL}}^{48}[\%]$ & 2.30 & 8.20 & 5.47 & 1.05 & 19.88 \\
$\mathrm{DL}_{96}[\%]$ & 0.98 & 16.47 & 5.86 & 2.57 & 47.15 \\
$\mathrm{DL}_{144}[\%]$ & 1.52 & 18.61 & 8.40 & 2.91 & 37.66 \\
$\mathrm{TY}^{2}[\%]$ & 3.03 & 19.94 & 11.00 & 2.94 & 29.65 \\
$\mathrm{WBSF}_{48}\left[\mathrm{~N} / \mathrm{cm}^{2}\right]$ & 76.33 & 114.72 & 103.69 & 6.05 & 5.21 \\
$\mathrm{WBSF}_{144}\left[\mathrm{~N} / \mathrm{cm}^{2}\right]$ & 4.28 & 8.84 & 6.71 & 1.09 & 16.02 \\
& 2.58 & 9.07 & 6.97 & 1.43 & 21.85 \\
\hline
\end{tabular}

According to Mörlein et al. (2007a) $\mathrm{EC}_{24}$ could be successfully used to sort carcasses and therefore to decrease drip loss in pork (choosing carcasses with lowered drip loss for culinary purposes). However, in present study Pearson's correlations between $\mathrm{EC}_{24}$ and $\mathrm{DL}_{48}, \mathrm{DL}_{96}$ and $\mathrm{DL}_{144}$ were very low $(0.10,0.01$ and -0.07 respectively) - (Table 3). Also, in study of Jukna et al. (2012) $\mathrm{EC}_{24}$ was rather weakly associated with pork shear force, colour and water binding capacity $\left(\mathrm{r}=-0.34, \mathrm{r}=-0.19\right.$ and $\mathrm{r}=0.28$ respectively) with similar results noted for $\mathrm{EC}_{2}$. Cited authors suggested that proper carcass sorting on the basis of electrical conductivity could be obtained in pre-rigor state $\left(r=0.49\right.$ for $\mathrm{EC}_{45}$ and shear force and $\mathrm{r}=0.61$ for $\mathrm{EC}_{45}$ and water binding capacity). However, $\mathrm{r}$ value for $\mathrm{EC}_{45}$ and pork colour was still low (0.14). In our study Pearson's correlations between $\mathrm{EC}_{35}, \mathrm{EC}_{2}$ and $\mathrm{EC}_{3}$ and pork quality traits were much lower (the highest $r$ values, e.g. 0.30 were noted between $\mathrm{EC}_{2}$ and $\mathrm{a}^{*}$ and $\mathrm{EC}_{2}$ and $\left.\mathrm{DL}_{48}\right)$. Also, in study of Czyżak-Runowska $\boldsymbol{e}$ al. (2010) $\mathrm{EC}_{24}$ was rather weakly associated with WHC $\left(\mathrm{r}=0.344^{* * *}\right)$ and tenderness $(\mathrm{r}=0.1173)$. However, Mörlein et al $\mathbf{~ ( 2 0 0 7 b )}$ noted higher correlation between $\mathrm{EC}_{24}$ and $\mathrm{DL}_{48}$ in two groups (404 animals total) of (German Large White $\times$ German Landrace $) \times$ Piétrain fatteners $(\mathrm{r}=0.52$ and $\mathrm{r}=0.59$ respectively). In study of Karamucki et al. (2015) r values between $\mathrm{EC}_{48}$ and $\mathrm{L}^{*} \mathrm{a}^{*} \mathrm{~b}^{*}$ and WHC were $0.211^{*}, 0.258^{*}, 0.442^{* *}$ and $-0.338^{*}$ respectively. These values are higher than those obtained in present study but late time of measurement, e.g. 48 hours after slaughter limits its use for directly abattoirs purposes.

Table 3 Pearson's correlations between electrical conductivity measured up to 24 hours post mortem and pork quality traits.

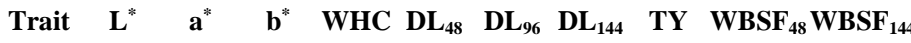

\begin{tabular}{lcccccccccc}
\hline $\mathrm{EC}_{35}$ & 0.10 & 0.13 & -0.03 & -0.04 & 0.24 & 0.09 & 0.06 & -0.28 & -0.19 & -0.18 \\
$\mathrm{EC}_{2}$ & 0.06 & $0.30^{*}$ & 0.13 & 0.07 & $0.30^{*}$ & 0.07 & -0.02 & -0.07 & -0.09 & -0.27 \\
$\mathrm{EC}_{3}$ & 0.22 & 0.16 & 0.09 & 0.13 & 0.21 & -0.02 & -0.07 & -0.10 & -0.09 & -0.25 \\
$\mathrm{EC}_{24}$ & 0.14 & 0.09 & 0.09 & 0.06 & 0.10 & -0.00 & -0.07 & -0.16 & 0.13 & 0.14
\end{tabular}

* - statistically significant at $\mathrm{P} \leq 0.05$

According to Joo et al. (2000) if only single predictor is used, the risk of incorrect pork evaluation occurs. Additionally, the potential of being able to predict broader quality description of pork could be advantageous (Kauffmann et al., 1993) Therefore, canonical analysis correlation including single and multiple electrical conductivity measurements with pork quality attributes was performed. Canonical correlations $\left(\mathrm{C}_{\mathrm{r}}\right)$ and square canonical correlations $\left(\mathrm{C}_{\mathrm{r}}^{2}\right)$ between single electrica conductivity measurements $\left(\mathrm{X}_{1}-\mathrm{X}_{4}\right)$ and sets of pork quality traits $\left(\mathrm{Y}_{1}-\mathrm{Y}_{10}\right)$ were presented in Table 4 . The highest $\mathrm{C}_{\mathrm{r}}$ and $\mathrm{C}_{\mathrm{r}}^{2}$ values were obtained for $\mathrm{EC}_{2}\left(\mathrm{X}_{2}\right)$ and $\mathrm{EC}_{3}\left(\mathrm{X}_{3}\right)$ with higher values between $\mathrm{X}_{2}$ and $\mathrm{Y}_{2}$ and $\mathrm{X}_{2}$ and $\mathrm{Y}_{3}$ sets (both $0.54^{* *}$ and 0.29 respectively). $\mathrm{C}_{\mathrm{r}}$ and $\mathrm{C}_{\mathrm{r}}^{2}$ values between $\mathrm{X}_{1}\left(\mathrm{EC}_{35}\right)$ and almost all sets of pork quality traits $\left(\mathrm{Y}_{2}-\mathrm{Y}_{10}\right)$ were low. The only exception was slightly higher values noted for $\mathrm{X}_{1}$ and $\mathrm{Y}_{1}\left(\mathrm{C}_{\mathrm{r}}=0.40^{*}\right.$ and $\mathrm{C}_{\mathrm{R}}{ }^{2}=0.16$ respectively). In study of Morel et al. (2006) $\mathrm{R}^{2}$ value for $\mathrm{EC}_{3}$ and drip loss measured 72 hours after slaughter was 0.34 Abovementioned authors found higher $\mathrm{R}^{2}$ between $\mathrm{EC}_{6}$ and $\mathrm{EC}_{24}$ and cooking loss (0.72 and 0.70, respectively) however lower $\mathrm{R}^{2}$ for $\mathrm{EC}_{24}$ and WBSF (below 0.20) No statistically significant $\mathrm{C}_{\mathrm{r}}$ and $\mathrm{C}_{\mathrm{r}}^{2}$ values between $\mathrm{EC}_{24}\left(\mathrm{X}_{4}\right)$ and all analysed sets of pork quality traits induced its exclusion from further examination.

Table 4 Canonical correlations $\left(\mathrm{C}_{\mathrm{r}}\right)$ and square canonical correlations $\left(\mathrm{C}_{\mathrm{r}}^{2}\right)$ between single electrical conductivity measurements $\left(\mathrm{X}_{1}-\mathrm{X}_{4}\right)$ and sets of pork quality traits $\left(\mathrm{Y}_{1}-\mathrm{Y}_{10}\right)$.

\begin{tabular}{|c|c|c|c|c|c|}
\hline \multirow{2}{*}{\multicolumn{2}{|c|}{ Dependent variables (Y) }} & \multicolumn{4}{|c|}{ Independent variables $(\mathrm{X})$} \\
\hline & & \multirow{2}{*}{$\frac{\mathrm{X}_{1}-\mathrm{EC}_{35}}{0.40^{*}}$} & \multirow{2}{*}{$\frac{\mathrm{X}_{2}-\mathrm{EC}_{2}}{0.47^{* *}}$} & \multirow{2}{*}{$\frac{\mathrm{X}_{3}-\mathrm{EC}_{3}}{0.47^{* *}}$} & \multirow{2}{*}{$\frac{\mathrm{X}_{4}-\mathrm{EC}_{24}}{0.25^{\mathrm{NS}}}$} \\
\hline & $\mathrm{C}_{\mathrm{r}}$ & & & & \\
\hline $\mathrm{Y}_{1}-\mathrm{L}, \mathrm{a}, \mathrm{b}$ & $\mathrm{C}_{\mathrm{r}}^{2}$ & 0.16 & 0.22 & 0.22 & 0.06 \\
\hline $\mathrm{Y}_{2}-\mathrm{DL}_{48}, \mathrm{DL}_{96}$ & $\mathrm{C}_{\mathrm{r}}$ & $0.35^{\mathrm{NS}}$ & $0.54^{* *}$ & $0.49^{* *}$ & $0.27^{\mathrm{NS}}$ \\
\hline $\mathrm{DL}_{144}$ & $\mathrm{C}_{\mathrm{r}}^{2}$ & 0.12 & 0.29 & 0.23 & 0.07 \\
\hline $\mathrm{Y}_{3}-\mathrm{DL}_{48}, \mathrm{DL}_{96}$ & $\mathrm{C}_{\mathrm{r}}$ & $0.36^{\mathrm{NS}}$ & $0.54^{* *}$ & $0.49^{* *}$ & $0.28^{\mathrm{NS}}$ \\
\hline $\mathrm{DL}_{144}, \mathrm{WHC}$ & $\mathrm{C}_{\mathrm{r}}^{2}$ & 0.13 & 0.29 & 0.24 & 0.07 \\
\hline $\mathrm{Y}_{4}-\mathrm{WBSF}_{48}$ & $\mathrm{C}_{\mathrm{r}}$ & $0.23^{\mathrm{NS}}$ & $0.27^{\mathrm{NS}}$ & $0.25^{\mathrm{NS}}$ & $0.16^{\mathrm{NS}}$ \\
\hline $\mathrm{WBSF}_{144}$ & $\mathrm{C}_{\mathrm{r}}^{2}$ & 0.05 & 0.07 & 0.06 & 0.03 \\
\hline $\mathrm{Y}_{5}-\mathrm{DL}_{48}$ & $\mathrm{C}_{\mathrm{r}}$ & $0.28^{\mathrm{NS}}$ & $0.31^{\mathrm{NS}}$ & $0.22^{\mathrm{NS}}$ & $0.19^{\mathrm{NS}}$ \\
\hline $\mathrm{WBSF}_{48}$ & $\mathrm{C}_{\mathrm{r}}^{2}$ & 0.08 & 0.09 & 0.05 & 0.03 \\
\hline $\mathrm{Y}_{6}-\mathrm{DL}_{48}, \mathrm{WHC}$ & $\mathrm{C}_{\mathrm{r}}$ & $0.30^{\mathrm{NS}}$ & $0.31^{\mathrm{NS}}$ & $0.23^{\mathrm{NS}}$ & $0.19^{\mathrm{NS}}$ \\
\hline $\mathrm{WBSF}_{48}$ & $\mathrm{C}_{\mathrm{r}}^{2}$ & 0.09 & 0.09 & 0.05 & 0.04 \\
\hline $\mathrm{Y}_{7}-\mathrm{DL}_{48}, \mathrm{WHC}$ & $\mathrm{C}_{\mathrm{r}}$ & $0.31^{\mathrm{NS}}$ & $0.31^{\mathrm{NS}}$ & $0.29^{\mathrm{NS}}$ & $0.22^{\mathrm{NS}}$ \\
\hline $\mathrm{WBSF}_{48}, \mathrm{~L}^{*}$ & $\mathrm{C}_{\mathrm{r}}^{2}$ & 0.10 & 0.09 & 0.08 & 0.05 \\
\hline $\mathrm{Y}_{8}-\mathrm{DL}_{48}, \mathrm{WHC}$ & $\mathrm{C}_{\mathrm{r}}$ & $0.35^{\mathrm{NS}}$ & $0.31^{\mathrm{NS}}$ & $0.23^{\mathrm{NS}}$ & $0.18^{\mathrm{NS}}$ \\
\hline TY & $\mathrm{C}_{\mathrm{r}}^{2}$ & 0.12 & 0.09 & 0.05 & 0.03 \\
\hline $\mathrm{Y}_{9}-\mathrm{DL}_{48}, \mathrm{WHC}$ & $\mathrm{C}_{\mathrm{r}}$ & $0.35^{\mathrm{NS}}$ & $0.31^{\mathrm{NS}}$ & $0.28^{\mathrm{NS}}$ & $0.20^{\mathrm{NS}}$ \\
\hline TY, L* & $\mathrm{C}_{\mathrm{r}}^{2}$ & 0.12 & 0.09 & 0.08 & 0.04 \\
\hline $\mathrm{Y}_{10}-\mathrm{DL}_{48}, \mathrm{WHC}$ & $\mathrm{C}_{\mathrm{r}}$ & $0.36^{\mathrm{NS}}$ & $0.31^{\mathrm{NS}}$ & $0.24^{\mathrm{NS}}$ & $0.26^{\mathrm{NS}}$ \\
\hline $\mathrm{TY}, \mathrm{L}^{*}, \mathrm{WBSF}_{48}$ & $\mathrm{C}_{\mathrm{r}}^{2}$ & 0.13 & 0.09 & 0.06 & 0.07 \\
\hline
\end{tabular}

In present survey conjunction of different electrical conductivity measurements only slightly rise determination of pork quality attributes sets (Table 5). The highest $C_{r}$ and $C_{r}^{2}$ values were noted between $X_{8}$ and $Y_{2}$ and $X_{8}$ and $Y_{3}$ (both $0.57^{* * *}$ and 0.32 respectively). However, more favourable measurement for direct abattoir use was $\mathrm{X}_{2}\left(\mathrm{EC}_{2}\right)$ due to similar $\mathrm{C}_{\mathrm{r}}$ and $\mathrm{C}_{\mathrm{R}}^{2}$ values with $\mathrm{Y}_{2}$ and $\mathrm{Y}_{3}$ (both $0.54^{* *}$ and 0.29 ) with only one measurement needed (in comparison to three for $X_{8}$ ). In production conditions at the abattoirs the tendency of decreasing the number of 
different measurements is common and desirable. However, $\mathrm{X}_{2}$ set was rather weakly associated with remaining pork quality attributes $\left(\mathrm{Y}_{4}-\mathrm{Y}_{10}\right)$ with exception for $Y_{1}$ set $\left(C_{r}\right.$ and $C_{R}^{2}$ values of $0.47^{* *}$ and 0.22 respectively) which reduces its utilisation ability. Although it has to be mentioned that in study of Tarczyński $\boldsymbol{e t}$ al. (2018) $\mathrm{EC}_{2}$ was useful in the same extent in pork quality classes assessment as $\mathrm{pH}$ measured 45 min after slaughter. In study of Morel et al. (2006) $\mathrm{R}^{2}$ for $\mathrm{EC}_{3}$ and set of four pork quality traits, e.g. $\mathrm{DL}_{72}$, cooking loss, WBSF and colour was 0.631 .

Table 5 Canonical correlations $\left(\mathrm{C}_{\mathrm{r}}\right)$ and square canonical correlations $\left(\mathrm{C}_{\mathrm{r}}^{2}\right)$ between multiple electrical conductivity measurements $\left(\mathrm{X}_{5}-\mathrm{X}_{8}\right)$ and sets of pork quality traits $\left(\mathrm{Y}_{1}-\mathrm{Y}_{10}\right)$

\begin{tabular}{|c|c|c|c|c|c|}
\hline \multirow{2}{*}{\multicolumn{2}{|c|}{$\begin{array}{l}\text { Dependent variables } \\
\text { (Y) }\end{array}$}} & \multicolumn{4}{|c|}{ Independent variables $(\mathrm{X})$} \\
\hline & & \multirow{2}{*}{$\begin{array}{c}\mathrm{X}_{5}-\mathrm{EC}_{35}, \\
\mathrm{EC}_{2} \\
0.47^{*}\end{array}$} & \multirow{2}{*}{$\begin{array}{c}\mathrm{X}_{6}-\mathrm{EC}_{35}, \\
\mathrm{EC}_{3} \\
0.47^{*}\end{array}$} & \multirow{2}{*}{$\begin{array}{c}\mathrm{X}_{7}-\mathrm{EC}_{2}, \\
\mathrm{EC}_{3} \\
0.49^{*}\end{array}$} & \multirow{2}{*}{$\begin{array}{c}\mathrm{X}_{8}-\mathrm{EC}_{35}, \\
\mathrm{EC}_{2}, \mathrm{EC}_{3} \\
0.49^{*}\end{array}$} \\
\hline & $\mathrm{C}_{\mathrm{r}}$ & & & & \\
\hline $\mathrm{Y}_{1}-\mathrm{L}, \mathrm{a}, \mathrm{b}$ & $\mathrm{C}_{\mathrm{r}}^{2}$ & 0.23 & 0.22 & 0.24 & 0.24 \\
\hline $\mathrm{Y}_{2}-\mathrm{DL}_{48}$ & $\mathrm{C}_{\mathrm{r}}$ & $0.55^{* *}$ & $0.48^{*}$ & $0.55^{* *}$ & $0.57^{* *}$ \\
\hline $\mathrm{DL}_{96}, \mathrm{DL}_{144}$ & $\mathrm{C}_{\mathrm{r}}^{2}$ & 0.30 & 0.23 & 0.30 & 0.32 \\
\hline $\mathrm{Y}_{3}-\mathrm{DL}_{48}$ & $\mathrm{C}_{\mathrm{r}}$ & $0.55^{* *}$ & $0.50^{*}$ & $0.55^{* *}$ & $0.57^{* *}$ \\
\hline $\begin{array}{l}\text { DL }_{96}, \text { DL }_{144} \\
\text { WHC }\end{array}$ & $\mathrm{C}_{\mathrm{r}}^{2}$ & 0.30 & 0.25 & 0.30 & 0.32 \\
\hline $\mathrm{Y}_{4}-\mathrm{WBSF}_{48}$ & $\mathrm{C}_{\mathrm{r}}$ & $0.27^{\mathrm{NS}}$ & $0.25^{\mathrm{NS}}$ & $0.28^{\mathrm{NS}}$ & $0.29^{\mathrm{NS}}$ \\
\hline $\mathrm{WBSF}_{144}$ & $\mathrm{C}_{\mathrm{r}}^{2}$ & 0.07 & 0.06 & 0.08 & 0.09 \\
\hline $\mathrm{Y}_{5}-\mathrm{DL}_{48}$ & $\mathrm{C}_{\mathrm{r}}$ & $0.36^{\mathrm{NS}}$ & $0.28^{\mathrm{NS}}$ & $0.31^{\mathrm{NS}}$ & $0.31^{\mathrm{NS}}$ \\
\hline $\mathrm{WBSF}_{48}$ & $\mathrm{C}_{\mathrm{r}}^{2}$ & 0.16 & 0.08 & 0.09 & 0.10 \\
\hline $\mathrm{Y}_{6}-\mathrm{DL}_{48}$ & $\mathrm{C}_{\mathrm{r}}$ & $0.36^{\mathrm{NS}}$ & $0.32^{\mathrm{NS}}$ & $0.31^{\mathrm{NS}}$ & $0.33^{\mathrm{NS}}$ \\
\hline $\begin{array}{l}\mathrm{WHC} \\
\mathrm{WBSF}_{48}\end{array}$ & $\mathrm{C}_{\mathrm{r}}^{2}$ & 0.13 & 0.10 & 0.10 & 0.11 \\
\hline $\begin{array}{l}\mathrm{Y}_{7}-\mathrm{DL}_{48}, \\
\mathrm{WHC}\end{array}$ & $\mathrm{C}_{\mathrm{r}}$ & $0.38^{\mathrm{NS}}$ & $0.32^{\mathrm{NS}}$ & $0.33^{\mathrm{NS}}$ & $0.34^{\mathrm{NS}}$ \\
\hline $\mathrm{WBSF}_{48}, \mathrm{~L}^{*}$ & $\mathrm{C}_{\mathrm{r}}^{2}$ & 0.14 & 0.10 & 0.11 & 0.12 \\
\hline $\mathrm{Y}_{8}-\mathrm{DL}_{48}$ & $\mathrm{C}_{\mathrm{r}}$ & $0.37^{\mathrm{NS}}$ & $0.39^{\mathrm{NS}}$ & $0.31^{\mathrm{NS}}$ & $0.39^{\mathrm{NS}}$ \\
\hline WHC, TY & $\mathrm{C}_{\mathrm{r}}^{2}$ & 0.13 & 0.15 & 0.10 & 0.15 \\
\hline $\mathrm{Y}_{9}-\mathrm{DL}_{48}$ & $\mathrm{C}_{\mathrm{r}}$ & $0.37^{\mathrm{NS}}$ & $0.40^{\mathrm{NS}}$ & $0.33^{\mathrm{NS}}$ & $0.40^{\mathrm{NS}}$ \\
\hline WHC, TY, L ${ }^{*}$ & $\mathrm{C}_{\mathrm{r}}^{2}$ & 0.14 & 0.16 & 0.11 & 0.16 \\
\hline $\mathrm{Y}_{10}-\mathrm{DL}_{48}$ & $\mathrm{C}_{\mathrm{r}}$ & $0.39^{\mathrm{NS}}$ & $0.39^{\mathrm{NS}}$ & $0.31^{\mathrm{NS}}$ & $0.41^{\mathrm{NS}}$ \\
\hline $\begin{array}{l}\mathrm{WHC}, \mathrm{TY}, \\
\mathrm{L}^{*}, \mathrm{WBSF}_{48}\end{array}$ & $\mathrm{C}_{\mathrm{r}}^{2}$ & 0.15 & 0.15 & 0.10 & 0.17 \\
\hline
\end{tabular}

Legend: ${ }^{* *}$ - statistically significant at $\mathrm{P} \leq 0.05,{ }^{*}$ - statistically significant at $\mathrm{P} \leq 0.05$

Table 6 Correlation between canonical variables U (electrical conductivity measured up to 24 hours post mortem) and $\mathrm{V}\left(\mathrm{L}^{*}, \mathrm{a}^{*}, \mathrm{~b}^{*}\right.$, drip loss measured up to 144 hours post mortem, $\mathrm{WHC} \mathrm{WBSF}_{48}$ and $\left.\mathrm{WBSF}_{144}\right)$

\begin{tabular}{lcccc}
\hline Traits & \multicolumn{4}{c}{ Canonical variables } \\
\hline Variables explaining & $\mathrm{U} 1$ & $\mathrm{U} 2$ & $\mathrm{U} 3$ & $\mathrm{U} 4$ \\
other variables & 0.69 & -0.67 & 0.21 & -0.18 \\
EC $_{35}$ & 0.94 & -0.03 & 0.17 & -0.29 \\
$\mathrm{EC}_{2}$ & 0.82 & -0.20 & 0.45 & 0.29 \\
$\mathrm{EC}_{3}$ & 0.26 & -0.27 & 0.87 & -0.32 \\
EC $_{24}$ & $\mathrm{~V} 1$ & $\mathrm{~V} 2$ & $\mathrm{~V} 3$ & $\mathrm{~V} 4$ \\
Explained variables & 0.12 & -0.12 & 0.39 & 0.55 \\
$\mathrm{~L}^{*}$ & 0.40 & 0.27 & 0.01 & -0.42 \\
$\mathrm{a}^{*}$ & 0.16 & 0.42 & 0.27 & -0.09 \\
$\mathrm{~b}^{*}$ & 0.42 & -0.07 & -0.04 & -0.28 \\
DL $_{48}$ & 0.07 & -0.15 & -0.19 & -0.35 \\
DL $_{96}$ & -0.02 & -0.27 & -0.31 & -0.18 \\
DL $_{144}$ & 0.12 & 0.30 & 0.28 & 0.30 \\
HHC $_{\text {WBSF }}$ & -0.53 & -0.01 & 0.46 & -0.43 \\
WBSF $_{144}$ & -0.23 & 0.43 & 0.51 & -0.23 \\
\hline
\end{tabular}

In present study canonical variables $\mathrm{U} 1$ and $\mathrm{V} 1$ were connected with $\mathrm{EC}_{2}$ and $\mathrm{EC}_{3}$ to high extent, e.g. 0.94 and 0.82 respectively (Table 6). This was in accordance with the highest $\mathrm{C}_{\mathrm{r}}$ and $\mathrm{C}_{\mathrm{r}}^{2}$ values noted between sets containing this parameters and $\mathrm{Y}_{2}$ and $\mathrm{Y}_{3}$ sets (Table 4 and 5). Canonical variables $\mathrm{U}_{2}$ and $\mathrm{V}_{2}$ were connected to highest extent with $\mathrm{EC}_{35}(-0.67), \mathrm{U} 3$ and V3 with $\mathrm{EC}_{24}(0.87)$ and $\mathrm{U} 4$ and V4 with $\mathrm{L}^{*}(0.55)$.

\section{CONCLUSION}

In this study weak Pearson's correlations between electrical conductivity measured up to 24 hours post mortem and pork quality traits were found. Moreover, on the basis of canonical correlation analysis it could also be presumed that its single and multiple measurements are not an useful predictor of pork quality attributes. The only exception (but only to some extent) was its potential in drip loss and water holding capacity assessment. This study should also be considered as preliminary because of its limited population (additional examination should be carried on much larger number of fatteners) however potential revision of the electrical conductivity use in pork quality evaluation should be taken into account.

\section{REFERENCES}

Czyżak-Runowska, G., Łyczyński, A., Pospiech, E., Koćwin-Podsiadła, M. Wojtczak, J., Rzosińska, E., ... \& Sieczkowska, H. (2010). Przewodnośc elektryczna mięsa wieprzowego jako wskaźnik jego jakości. Journal of Central European Agriculture, 11(1), 105-111.DOI: /10.5513/JCEA01/11.1.826

Dokmanovic, M., Baltic, M. Z., Duric, J., Ivanovic, J., Popovic, L., Todorovic, M. ... \& Pantic, S. (2015). Correlations among stress parameters, meat and carcass quality parameters in pigs. Asian-Australasian Journal of Animal Sciences, 28(3), 435.https://doi.org/10.5713/ajas.14.0322

Font-i-Furnols, M., \& Guerrero, L. (2014). Consumer preference, behavior and perception about meat and meat products: An overview. Meat science, 98(3), 361371.https://doi.org/10.1016/j.meatsci.2014.06.025

Grau, R., \& Hamm, R. (1952). Eine einfache methode zur bestimmung der wasserbindung in fleisch. Fleischwirtschaft, 4, 295-297.

Grunert, K. G., Verbeke, W., Kügler, J. O., Saeed, F., \& Scholderer, J. (2011). Use of consumer insight in the new product development process in the meat sector. Meat Science, 89(3), 251-258.https://doi.org/10.1016/j.meatsci.2011.04.024

Joo, S. T., Kauffman, R. G., Warner, R. D., Borggaard, C., Stevenson-Barry, J. M., Lee, S., ... \& Kim, B. C. (2000). Objectively predicting ultimate quality of postrigor pork musculature: I. Initial comparison of techniques. Asian-Australasian Journal of Animal Sciences, 13(1), 68-76.https://doi.org/10.5713/ajas.2000.68 Jukna, V., Jukna, Č., \& Pečiulaitienè, N. (2012). Electrical conductivity of pig meat and its relation with quality. Veterinarija ir zootechnika, 57(79), 18-21.

Karamucki, T., Rybarczyk, A., Jakubowska, M., \& Rybak, K. (2015). The quality of the longisimus lumborum muscle of pietrain-cross fatteners in relation to electrical conductivity 48 hours post mortem. Scientific Annals of Polish Society of Animal Production, 2(4), 75-83.

Kauffman, R. G., Sybesma, W., Smulders, F. J. M., Eikelenboom, G., Engel, B. Van Laack, R. L. J. M., ... \& Van der Wal, P. G. (1993). The effectiveness of examining early post-mortem musculature to predict ultimate pork quality. Meat Science, 34(3), 283-300.https://doi.org/10.1016/0309-1740(93)90078-V

Lee, S., Norman, J. M., Gunasekaran, S., Van Laack, R. L. J. M., Kim, B. C., \& Kauffman, R. G. (2000). Use of electrical conductivity to predict water-holding capacity in post-rigor pork. Meat Science, 55(4), 385 389.https://doi.org/10.1016/S0309-1740(99)00166-7

Lonergan, S. M., Huff-Lonergan, E., Rowe, L. J., Kuhlers, D. L., \& Jungst, S. B. (2001). Selection for lean growth efficiency in Duroc pigs influences pork quality. Journal of Animal Science, 79(8), 20752085.https://doi.org/10.2527/2001.7982075x

Morel, P. C. H., Camden, B. J., Purchas, R. W., \& Janz, J. A. M. (2006). Evaluation of three pork quality prediction tools across a 48 hours postmortem period. Asianaustralasian journal of animal sciences, 19(2), 266 272.https://doi.org/10.5713/ajas.2006.266

Mörlein, D., Link, G., Murani, E., Wimmers, K, \& Wicke, M. (2007a). Evaluation of three pig crossbreed types with respect to strategies to improve the meat quality: MHS genotype rather than crossbreed type influences drip loss. Archives Animal Breeding, 50(6), 605-618.https://doi.org/10.5194/aab-50-605-2007

Mörlein, D., Link, G., Werner, C., \& Wicke, M. (2007b). Suitability of three commercially produced pig breeds in Germany for a meat quality program with emphasis on drip loss and eating quality. Meat Science, 77(4), 504511.https://doi.org/10.1016/j.meatsci.2007.04.030

Ngapo, T. M., Martin, J. F., \& Dransfield, E. (2007). International preferences for pork appearance: I. Consumer choices. Food Quality and Preference, 18(1), 2636.https://doi.org/10.1016/j.foodqual.2005.07.001

Pliquett, F., Pliquett, U., \& Robekamp, W. (1990). Beurteilung der reifung des M. long. dorsi und M. semitendinosus durch impulsimpedanzmessungen. Fleischwirtschaft, 70(12), 1468-1470.

Pliquett, U., Altmann, M., Pliquett, F., \& Schöberlein, L. (2003). Py—a parameter for meat quality. Meat science, 65(4), 1429-1437.https://doi.org/10.1016/S03091740(03)00066-4

Pohja, M. S., \& Niinivaara, F. P. (1957). Die bestimmung der Wasserbindung des Fleisches mittels der Konstantdruckmethode. Fleischwirtschaft, 9, 193-195.

Prange, H., Ober, G., \& Jugert, L. (1977). Untersuchungen zur Muskelfleischqualitat beim Schwein. 2. Einfluss verschiedener Transportformen und Ausruhezeiten. Archiv fur experimentelle Veterinarmedizin.

Tarczyński, K., Sieczkowska, H., Zybert, A., Krzęcio-Nieczyporuk, E., \& Antosik, K. (2018). pH measured 24 hours post mortem should not be regarded as ultimate $\mathrm{pH}$ in pork meat quality evaluation. South African Journal of Animal Science, 48(6).http://dx.doi.org/10.4314/sajas.v48i6.2

Verbeke, W., Pérez-Cueto, F. J., de Barcellos, M. D., Krystallis, A., \& Grunert, K G. (2010). European citizen and consumer attitudes and preferences regarding beef and pork. Meat science, 284 292.https://doi.org/10.1016/j.meatsci.2009.05.001 
Weiss, D. J. (1972). Canonical correlation analysis in counseling psychology

research. Journal of Counseling Psychology, 19(3),

241.https://doi.org/10.1037/h0032675 